\title{
Eliciting clinicians, policymakers, patients and carers perspectives on implementation of technology based interventions for psychiatric patients in South Eastern Europe (SEE)
}

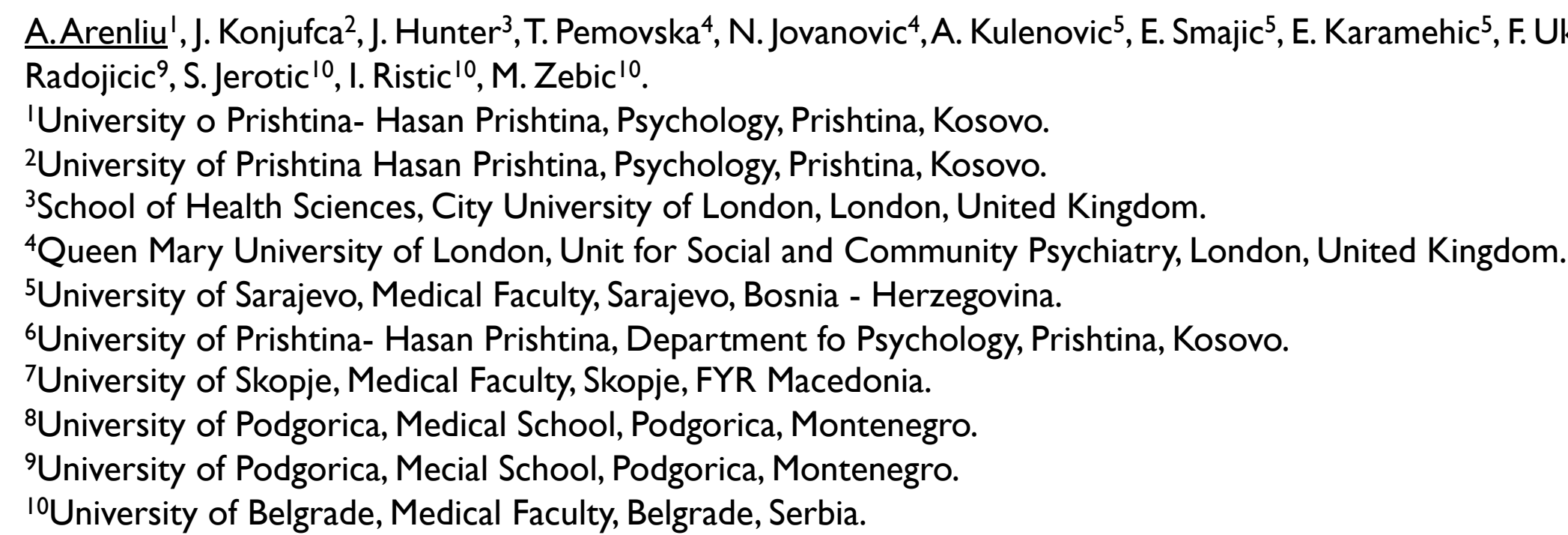

Objectives: Dialog Plus (Dialog + ) is computer assisted intervention designed to improve the quality of regular meetings between clinicians and patients with psychotic disorders by using solution focused approach, technology assisted approach to address issues with mental health, medication, physical health and overall personal and social functioning. In general, the intervention use tablets to better structure and improve quality of the interaction between patients and clinicians during therapeutical process. This study explores perspectives and attitudes of clinicians, policymakers, patients and caregivers on technology-assisted psychosocial intervention for patients with schizophrenia in 5 countries of SEE (Bosnia \& Herzegovina, Kosovo*, Montenegro, North-Macedonia and Serbia). Study is conducted prior to randomized clinical study to test the effectiveness of Dialog + in 5 countries.

Background: Psychotic disorders like schizophrenia are severe and complex mental health disorders that can cause a long-term disability. Schizophrenia represents a major health burden in low and middle-income countries (LMIC) often characterized with limited treatment resources. Implementation of a technologyassisted, low-cost intervention such as DIALOG+, which utilises existing personal and social resources in community, could help to lower the existing burden of schizophrenia in LMIC.

Materials and Methods: Study findings are based on data from 43 focus groups and 178 participants and total of (8 with clinicians, 8 with policymakers, 9 with patients and 7 with caregivers) conducted in 5 SEE countries In each focus group facilitators presented standardized DIALOG + presentation and asked perspectives of participants on DIALOG+. Data was coded and analysed using Atlas-Ti Software.

Results: The data analysis from focus groups identified in total 14 perceived potential difficulties such as , 2 I perceived potential gains (Table 2) and 36 perceived potential facilitators as grouped in Table I.

Perceived
potential
difficulties
Time pressure
clinicians have
(short visit time)
Limited human
resources and
average short time
spent with patients
Patients mental
health status might
not allow use of
app
Resistance to
change the old
patterns

Perceived
potential gains

Moving beyond used medical model

Better follow up of patients and continuous data collection

Focusing on problem solving

Empowers clients by making them more proactive

\section{Perceived \\ potential \\ facilitators}

Overall simplicity of application use Dialog +

Willingness of all patients, clinicians, caretakers and policymakers to use Dialog +

Consistency with previous norms and treatment policies

Willingness of majority of clinicians to try new treatments
Conclusions: Dialog + is perceived as feasible and welcomed by clinicians, policymakers, patients and carers. The findings also identified some potential difficulties in implementation of Dialog + which could be addressed prior to implementation study. Findings indicate some differences among populations with are worthwhile in implementation of the treatment. Study also briefly discusses the challenges of conducting qualitative study in multi-country studies. 